\title{
Time-Dependent Cellular Injuries Induced by Calcium Oxalate Monohydrate and Dihydrate Crystals
}

\author{
Jian-Min WANG, Ling-Hong HUANG and Jian-Ming OUYANG ${ }^{a, *}$ \\ Department of Chemistry, Jinan University, Guangzhou 510632, China \\ atoyjm@jnu.edu.cn \\ ${ }^{*}$ Corresponding author
}

Keywords: Calcium oxalate, time effect, cellular injury, renal epithelial cells.

\begin{abstract}
Objective: This study aims to compare the cytotoxicity and adhesion difference between oxalate monohydrate (COM) and dehydrate (COD) crystals and African green monkey kidney epithelial cells (Vero). Methods: Through the detection of cell viability, lactate dehydrogenase $(\mathrm{LDH})$ release amount, expression of hyaluronic acid, and propidium iodide staining method to compare the cell injury degree; scanning electron microscope was used to observe crystal adhesion. Results: Cellular damage increased as time extended within $1 \sim 12 \mathrm{~h}$. The cytotoxicity of COM was stronger than COD, and the adhesion amount of COM was much larger than COD. Conclusions: The formation of large amount of micron-sized COM crystals during short time in urine will produce cytotoxicity to kidney epithelial cells, which increasing the risk of stone formation, In contrast, the formation of COD cause relatively less cytotoxicity.
\end{abstract}

\section{Introduction}

Calcium oxalate $(\mathrm{CaOx})$ is the major crystalline component of kidney stones; and it mainly exists in two forms: calcium oxalate monohydrate (COM) and calcium oxalate dihydrate (COD) [1]. Meimeidou et al [2] confirmed that COM and COD crystals will produce free oxalic acid ions in cells, thus cause cell damaged.

The effect of crystals on cell properties has widely studied [3]. It was reported that the interaction time between cells and crystals is an important factor to affect cytotoxicity of crystals. For example, ultrafine $\mathrm{TiO}_{2}$ induced damage of human B-cell lymphoblastoid cell in a time-dependent manner in 6 48 h [4]. Ahamed et al [5] found that when human lung epithelial A549 cells were treated with nickel nanoparticle (Ni $\mathrm{NP}$ ) at the concentrations of $1,2,5,10$ and $25 \mu \mathrm{g} / \mathrm{ml}$ for 24 and $48 \mathrm{~h}$, Ni NPs could damage A549 in dose and time-dependent manner.

$\mathrm{CaOx}$ calculi formation is thought to follow two main routes, (slow) formation of subepithelial plaques at papillary tips and (rapid) formation of intratubular plugs [6]. Some kinds of stone formers make stones grow on Randall's plaques, an interstitial form of papillary calcification that does not involve the deposition of mineral in tubule lumens. Other kinds of stone formers produce mineral that lodges in the papillary collecting ducts, and these ductal plugs can also support the growth of stones that are attached to the papilla. These two mechanisms of stone retention are not mutually exclusive, as some patients who form stones on ductal plugs could also form stones on Randall's plaques, but there are certainly stone formers who have only one of these mechanisms and not the other. 
In this article, we comparatively studied of the effect of adhesion time of COM, COD crystals on Vero cells by detecting the change of biochemical indicators of cells and the adhesion amount of crystals.

\section{Experimental}

\section{Materials}

African green monkey renal epithelial (Vero) cells were purchased from Shanghai Cell Bank, Chinese Academy of Sciences (Shanghai, China).

Cell proliferation assay kit (CCK-8) was purchased from Dojindo Laboratories (Kumamoto, Japan). Lactate dehydrogenase (LDH) kit, biotinylated hyaluronic acid binding protein (bHABP) (MERCK Corporation, Germany), propidium iodide (PI), anti-fade fluorescence mounting medium and bovine serum albumin (BSA) were all purchased from Shanghai Beyotime Bio-Tech Co., Ltd. (Shanghai, China).

All conventional reagents used were analytically pure and purchased from Guangzhou Chemical Reagent Factory of China (Guangzhou, China).

\section{Apparatus}

X-L type environmental scanning electron microscope (SEM, Philips, Eindhoven, Netherlands), laser confocal microscope (LSM510 Meta Duo Scan, Zeiss, Jena, Germany), enzyme mark instrument (Safire2 ${ }^{\mathrm{TM}}$, Tecan, Männedorf, Switzerland), inductively coupled plasma emission spectrometer (ICP-AES, Optima 2000DV, Perkin Elmer, CT, USA) and flow cytometer (FACS Aria, BD Corporation, CA, USA).

\section{Experimental Methods}

\section{Preparation and Characterization of COM and COD Crystal}

COD and COM crystals were prepared as previously described [7], the products were characteristics by SEM and XRD.

\section{Cell Culture and Cell Viability Assay}

Vero cells were cultured in DMEM culture medium containing $10 \%$ fetal bovine serum in a $5 \% \mathrm{CO}_{2}$ humidified atmosphere at $37^{\circ} \mathrm{C}$. Cells were divided into 2 groups: (A) control group; (B) COD, COM crystals treatment group. Adhesion time is 1, 3, 6 and 12 hours respectively. The change of cell viability was measured using CCK-8 assay; lactate dehydrogenase was measured by LDH kid. Propidium iodide (PI) staining was used to observe cell death. HA expression assay were carried out according to reference [7].

\section{SEM Observation of HKC Treated By COD and COM Crystals}

After reaching the adhesion time, the supernatant was removed by suction, the left cell-crystal mixture was washed three times with PBS, fixed in $2.5 \%$ glutaraldehyde at $4{ }^{\circ} \mathrm{C}$ for $24 \mathrm{~h}$, fixed with $1 \% \mathrm{OsO} 4$, and dehydrated in gradient ethanol $(30 \%, 50 \%, 70 \%$, $90 \%$ and $100 \%$, respectively). After dried under the critical point of $\mathrm{CO} 2$, the cell-crystal mixture was treated with gold sputtering, and observed under SEM. 


\section{Results}

\section{Preparation and Characterization of Calcium Oxalate Microcrystalline}

Figs. 1a and 1b showed the SEM images of COM and COD crystals. COM crystals are mainly hexagonal and fork shape, COD crystals are tetragonal bipyramid, their average sizes were about $17 \mu \mathrm{m}$.

XRD patterns showed that diffraction peaks at $d=5.93,3.64,2.96$ and $2.35 \AA$, respectively, belonged to the $(\overline{101}),(020),(\overline{2} 02)$ and (130) crystal plane of COM crystals. The diffraction peaks at $\mathrm{d}=6.17,4.40,2.77$ and $2.24 \AA$ were belonged to the (200), (211), (411) and (213) crystal plane of COD crystals, respectively. These results showed that the synthetic crystals are COM and COD crystals.

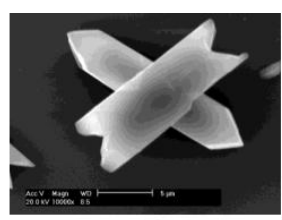

(a)

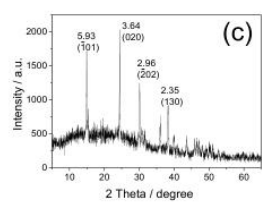

Fig. 1. SEM and XRD patterns of COM and COD crystals. (a,c) COM; (b,d) COD.

\section{Cell Viability Assay}

The viability of Vero cells after exposure to COM and COD crystals for different time was detected by CCK-8 assay (Fig. 2a). Both COM and COD have cytotoxicity to Vero, and the cytotoxicity was time-dependent. As the adhesion time increased from $3 \mathrm{~h}$ to 6 $\mathrm{h}, 12 \mathrm{~h}$, cell viability continues to reduce. COM showed higher toxicity than COD under same conditions. These results suggested that the formation of micron-sized COM crystal in urine would produce more toxicity to kidney epithelial cells than COD.

$\mathrm{LDH}$ is a stable enzyme of the cytoplasm that is released extracellularly once the cell membrane was ruptured. Thus, LDH is considered as a marker of cell membrane integrity. As shown in Fig. 2b, LDH release amount increased as interaction time extended, and COD crystals caused more LDH release than COM. This result is consistent with the cell viability results (Fig. 2a). That is, long time of crystal action will further damage Vero cells.
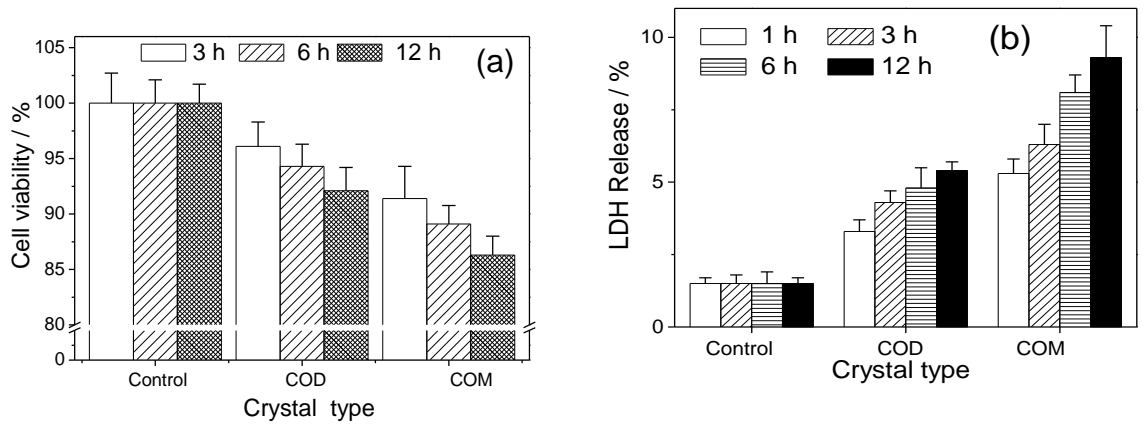

Fig. 2. Change in cell viability (a) and LDH release amount (b) of Vero cells after exposure to COM and COD crystals for different time. Crystal concentration: $200 \mu \mathrm{g} / \mathrm{mL}$. 


\section{Cell Death Detection by Propidium Iodide (PI) Staining}

PI is a commonly used fluorescent staining agent for the cell nucleus. PI cannot penetrate through normal cell membranes but can pass through terminal apoptotic cell and necrotic cell membranes and bind to DNA in the nucleus, thereby emitting red fluorescence [8]. The results of PI staining in Vero cells after interaction with COM and COD are shown in Fig. 3. At time $t=1 \mathrm{~h}$, only a small amount of nuclei were dyed red in the control group. However, the number of red-stained nuclei was evidently increased after the time increased. At $\mathrm{t}=12 \mathrm{~h}$ the maximum number of dyed nuclei were observed, that corresponded to the highest number of dead cells. The fluorescence enhancement caused by COD was smaller than COM crystal, which suggested that COM produce greater fatality rate to Vero cells than COD.

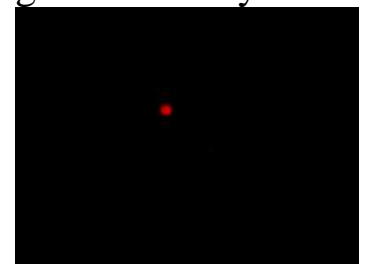

(a) Control-0 $\mathrm{h}$

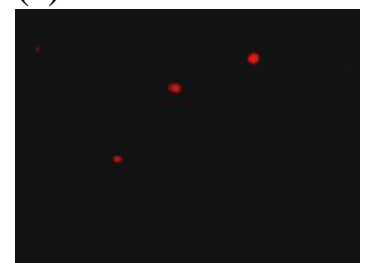

(b) COD-1 h;

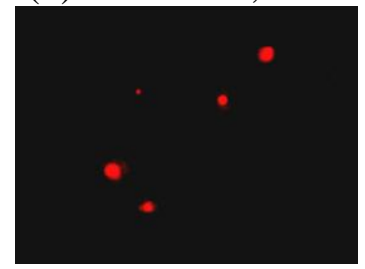

(f) $\mathrm{COM}-1 \mathrm{~h}$;

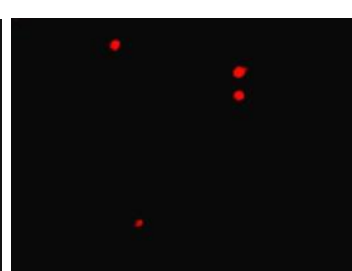

(c) COD-3 h;

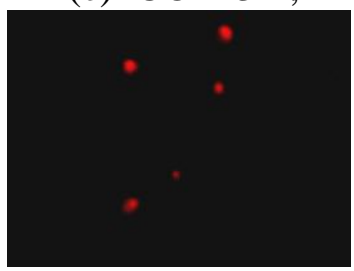

(g) COM-3 h;

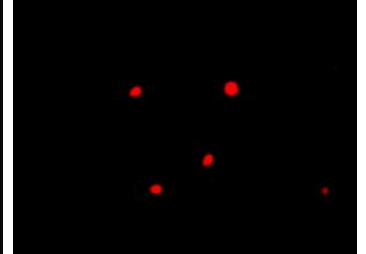

(d) COD-6 h;

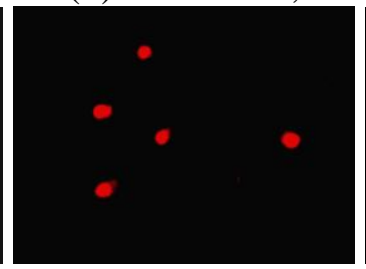

(h) COM-6 h;

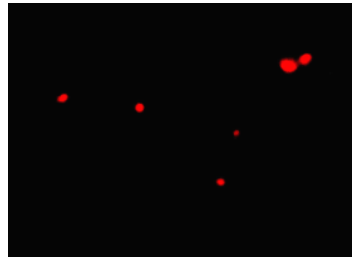

(e) COD-12 h

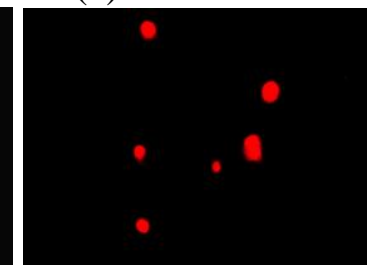

(i) $\mathrm{COM}-12 \mathrm{~h}$

Fig. 3. Fluorescence microscope $(\times 600)$ of PI staining results of Vero cells after exposure to COM and COD crystals for different time.

\section{Hyaluronan (HA) Expression Assay}

HA is not only a kind of cell matrix, but also one of the main adhesion molecules in cell membrane [9]. The HA expression of Vero after treated with COD and COM for different time were shown in Fig. 4. The green fluorescence increased as the crystal treat time increased, COM crystal-treated cells has stronger HA expression than COD, which indicates that COM induces more severe cell injury and greater HA expression.

\section{Crystal Adhesion Observed by SEM}

The adhesion between Vero cells and COM and COD crystals were detected by SEM (Fig. 5). There are more crystal adhesion on cell surface for COM crystals (Figs. 5c, 5c), and the aggregated COM crystals were also seen (Fig. 5c). By contrast, the adhesion and aggregation of COD crystals onVero were weak than COM (Figs. 5d, 5e). 


\section{Quantitative Determination of Crystal Adhesion}

In order to confirm the adhesion difference of COM and COD crystals on Vero surface, the crystal adhesion amount was measured by ICP method. As the adhesion time increases, the adhesion amount of crystals continued to increase. Under same conditions, the adhesive amount of COM is always higher than COD (Fig. 7).

\section{Conclusions}

The injury of Vero cells caused by COM, COD microcrystals increases with the increase of interaction time. After injured, cell viability reduces, LDH release amount increases, HA expression on cell surface increases, cell mortality increases, and crystal adhesion amount also increases. Under the same conditions, COM induces more severe cell injury than COD. It suggested that the formation of micron-sized COM crystals would cause toxicity to kidney epithelial cells, thus enhancing the risk of kidney stone formation.

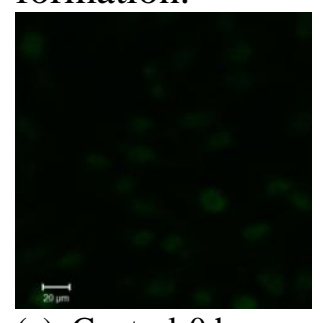

(a) Control-0 h
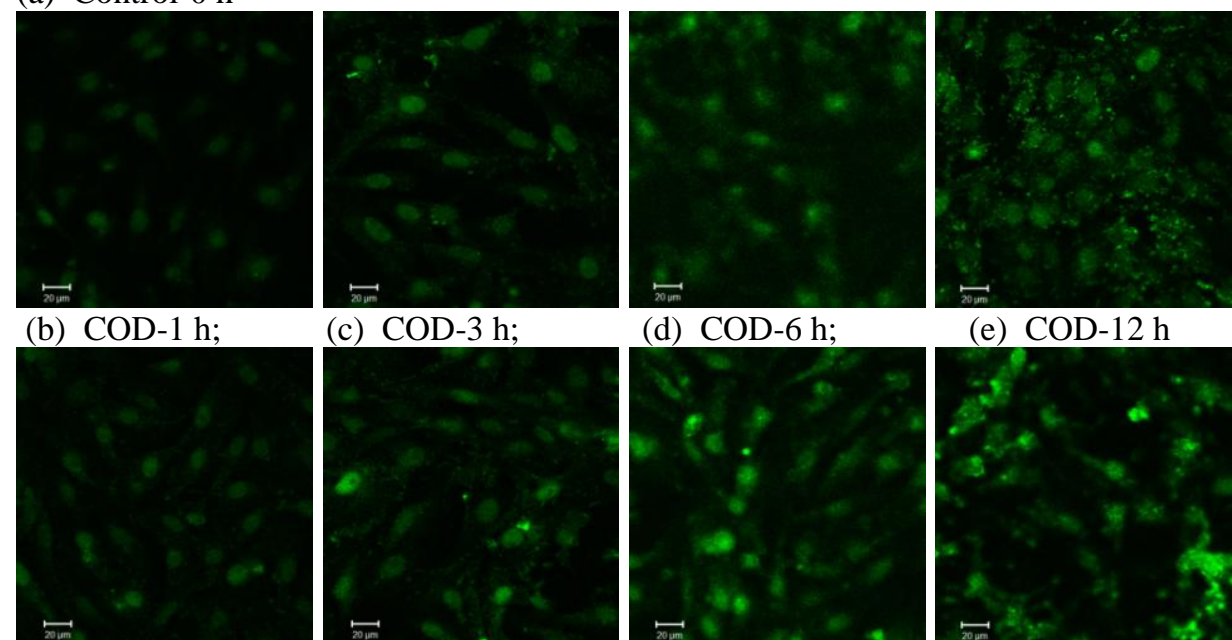

(d) COD-6 h;

(e) COD-12 h
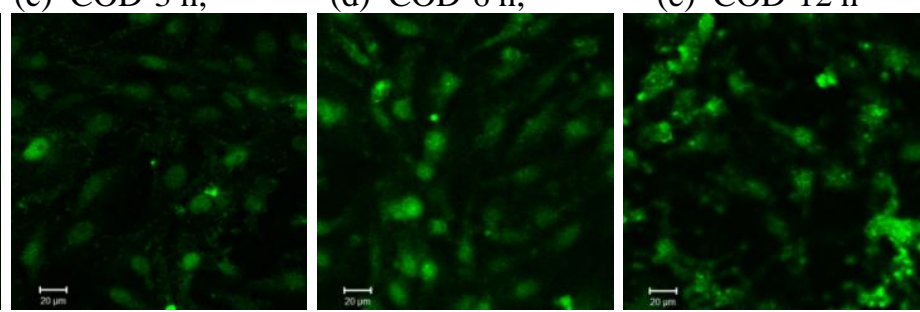

(f) COM-1 h;

(g) COM-3 h;

(h) COM-6 h;

(i) $\mathrm{COM}-12 \mathrm{~h}$

Fig. 4. Laser scanning confocal microscope images of HA expression of Vero cells after exposure to COM and COD crystals for different time.

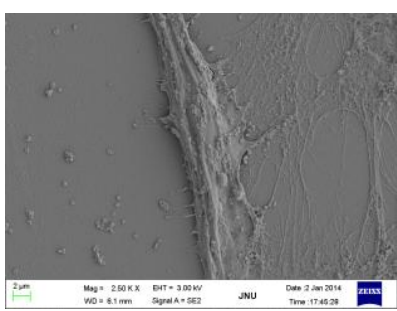

(a)

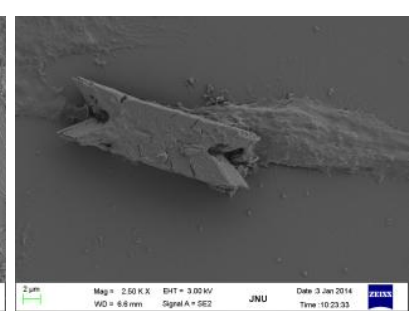

(b)

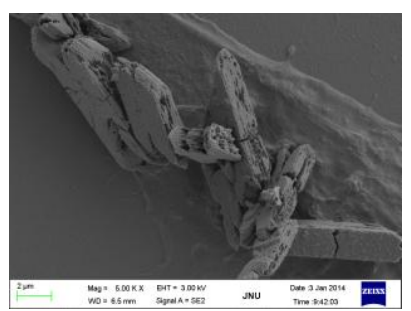

(c) 


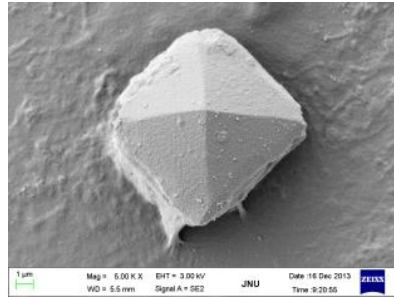

(d)

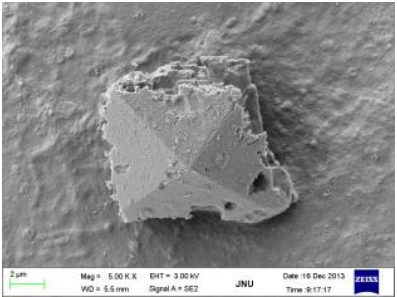

(e)

Fig. 5. SEM images of the control group without crystal (a), and Vero after exposure to COM (b,c) and COD crystals $(\mathrm{d}, \mathrm{e})$.

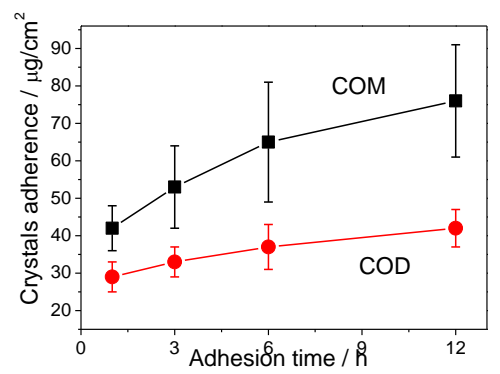

Fig. 6. Quantitative analysis of adhesion amount COM and COD crystals on Vero cells with adhesion time

\section{Acknowledgements}

This research work was granted by the Natural Science Foundation of China (No. 81670644).

\section{References}

[1] Farmanesh S, Ramamoorthy S, Chung J, Asplin J R, Karande P, Rimer J D. Specificity of growth inhibitors and their cooperative effects in calcium oxalate monohydrate crystallization. J. Am. Chem. Soc., 2014, 136: 367-376.

[2] Meimaridou E, Jacobson J, Seddon A M, Noronha-Dutra A A, Robertson W G, Hothersall J S. Crystal and microparticle effects on MDCK cell superoxide production: oxalate-specific mitochondrial membrane potential changes. Free Rad. Biol. Med., 2005, 38: 1553-1564.

[3] Yuen J W M, Gohel M-D I. The initial and subsequent inflammatory events during calcium oxalate lithiasis. Clin. Chim. Acta, 2010, 411: 1018-1026.

[4] Wang J J, Sanderson B J S, Wang H. Cyto-and genotoxicity of ultrafine TiO 2 particles in cultured human lymphoblastoid cells. Mutation Research/Genetic Toxicology and Environmental Mutagenesis, 2007, 628(2): 99-106.

[5] Ahamed M. Toxic response of nickel nanoparticles in human lung epithelial A549 cells. Toxicology in Vitro, 2011, 25(4): 930-936.

[6] Williams J C, McAter J A. Retention and growth of urinary stones: insights from imaging. J. Nephrol., 2013, 26: 25-31. 
[7] Sun X-Y, Ouyang J-M, Liu A-J, Ding Y-M, Li Y-B, Gan Q-Z. Preparation, characterization, and in vitro cytotoxicity of COM and COD crystals with various sizes. Mater. Sci. Eng. C, 2015, 57: 147-156.

[8] Schepers M S J, Van Ballegooijen E S, Bangma C H, Verkoelen C F. Crystals cause acute necrotic cell death in renal proximal tubule cells, but not in collecting tubule cells. Kidney Int., 2005, 68: 1543-1553.

[9] Poon N W, Gohel M D I, Urinary glycosaminoglycans and glycoproteins in a calcium oxalate crystallization system. Carbohyd. Res., 2012, 347: 64-68. 